\title{
Cancer Epidemiology in Lahore, Pakistan - 2010-2015
}

\author{
Farhana Badar1', Shahid Mahmood1', Mohammad Tariq Mahmood², Misbah Masood², Imrana Tanvir4, \\ Omar Rasheed Chughtai ${ }^{5}$, Shahida Niazi ${ }^{6}$ and Alia Ahmad ${ }^{7}$ \\ ${ }^{1}$ Cancer Registry and Clinical Data Management, Shaukat Khanum Memorial Cancer Hospital and Research Centre, Lahore, Pakistan \\ 2Department of Pathology, Shaukat Khanum Memorial Cancer Hospital and Research Centre, Lahore, Pakistan \\ ${ }^{3}$ Oncology Division, Institute of Nuclear Medicine and Oncology, Lahore, Pakistan \\ ${ }^{4}$ Department of Pathology, King Abdulaziz University, Jeddah, Saudi Arabia \\ ${ }^{5}$ Chughtai Lab, Lahore, Pakistan \\ ${ }^{6}$ Department of Pathology, King Edward Medical University, Lahore, Pakistan \\ ${ }^{7}$ Department of Pediatric Hematology/Oncology, The Children's Hospital and The Institute of Child Health, Lahore, Pakistan
}

\begin{abstract}
Objective: To study the cancer incidence rates in Lahore, which has an estimated annual population count of 10.3 million. Study Design: Cross-sectional study.

Place and Duration of Study: Data on new cancer cases diagnosed between 2010 and 2015, among the residents of Lahore district, Pakistan, was reviewed retrospectively in 2015-2017.

Methodology: Nineteen collaborating centres of the population-based Punjab Cancer Registry (PCR), representing both the government and private sectors, reported their cases to the coordinating office located within the Shaukat Khanum Memorial Cancer Hospital and Research Centre (SKMCH\&RC). The age-standardised incidence rates (ASIR) per 100,000 population, over a six-year period, were computed. Sixteen 5-year age groups were created beginning from 0-4 to 70-74 years, followed by $75+$ years. Graphs on the five-year age-specific incidence rates by gender, were also generated.

Results: Between 2010 and 2015, 33,028 new malignancies were recorded in Lahore, with the crude average annual incidence rate being 53.1. In adults, the highest ASIRs were noted for cancers of the breast (77.3) among females and of prostate (11.4) in men. Age-specific incidence rates for female breast cancer showed an upward trend at the age of 20 years, reaching a figure of 160 at the age of 55 years. Among males, the rates for prostate cancer started to increase at the age of 55 years and reached a peak of 93 at 75 years.

Conclusion: These results warrant expanding cancer registration in the region and sharing statistics with policy-makers to establish hospitals accordingly to manage cancer, along with exploring various risk factors within the population.
\end{abstract}

Key Words: Cancer incidence, Lahore, Malignancies, Population-based, Punjab Cancer Registry.

How to cite this article: Badar F, Mahmood S, Mahmood MT, Masood M, Tanvir I, Chughtai OR, Niazi S, Ahmad A. Cancer epidemiology In Lahore, Pakistan - 2010-2015. J Coll Physicians Surg Pak 2020; 30(2):113-122.

\section{INTRODUCTION}

Pakistan is categorised as a lower-middle-income country, with population estimated at 197 million in 2017 by the World Bank. ${ }^{1}$ Although Pakistan is a populous country, there is a dearth of oncologists or dedicated facilities that deal specifically with cancer diagnosis or management. ${ }^{2}$ This is compounded by the fact that cancer registration has never been taken seriously in the country in more than 70 years of existence, and enough efforts have not been made to establish populationbased cancer registries in the region. Except for the population-based data from the Karachi Cancer Registry

Correspondence to: Dr. Farhana Badar, Cancer Registry and Clinical Data Management, Shaukat Khanum Memorial Cancer Hospital and Research Centre, 7-A, Block R-3, Johar Town, Lahore 54782, Pakistan

E-mail:farhana@skm.org.pk

Received: September 11, 2019; Revised: January 13, 2020;

Accepted: January 13, 2020
$(\mathrm{KCR}),{ }^{3}$ which was published by the International Agency for Research on Cancer (IARC) in 2007, the data reported from few other centres is institutional and does not represent the population of the region. Even the KCR data represented merely 1.7 million population of the Karachi South district, accounting for nearly $1 \%$ of the population of the country. The government's total expenditure on health is $2.6 \%$ of the GDP, and healthcare delivery is quite complex, with a large part of the population being served through a mixed health system via multiple health providers. ${ }^{4}$

The Punjab Cancer Registry was established in 2005 and the reporting of cancer cases was initiated under a mutual agreement between various centres. ${ }^{5}$ During the early phase of the Registry, enough information could not be collected. Later, reports on its 3-year data (20102012) were published.6,7 The current study is a comprehensive, retrospective study over an extended six-year period (2010-2015) reporting the cancer incidence rates within the population of Lahore. 
The objective was to determine the incidence rates for the cancers reported according to age group and present the age-specific incidence rates for cancers commonly diagnosed in the provincial metropolis.

\section{METHODOLOGY}

A population-based study was conducted in the district of Lahore, which is located in the northeast region of Pakistan. Its total land area is 1,772 square kilometres, with its average population density being 5,551 persons, per square kilometre. 8 The population of Lahore was taken as the population denominator for computing the incidence rates using cancer counts as the numerator. The average annual population estimate, determined using an average annual growth rate of $3.46 \%$ for Lahore, ${ }^{9}$ over six years, was $10,364,878$.

The 19 Punjab Cancer Registry (PCR) collaborating centres represent hospitals, laboratories, and collection centres, both in the government and private sectors, of the metropolis. Each centre is assigned a specific identification number within the Registry. The Registry also allocates a case record number, while retaining the specific number assigned to the case by the collaborating service. Data sources include the medical records from the clinical areas as the inpatient admissions, outpatient/ emergency assessment visits, hospital death certificates, pathology/operative reports, chemotherapy/radiotherapy logs, radiology/nuclear medicine reports, and walk-in clinic logs (the initial assessment clinics where patients are triaged). In this way, information on patients, who are not registered through the walk-in clinics after undergoing initial assessment, is also captured into the database. Such patients go elsewhere for treatment, perhaps to another city, or opt for alternative forms of treatment. A check for duplicate registration is conducted using various combinations of name/age/gender/phone number/address/tumour morphology; and once it has been identified that a patient has been registered twice, they are included just once in the report.

Data are captured using both the active and passive methods of data collection and then entered into the system. Data collected by the data collectors of 19 centres within Lahore on the prescribed data collection form are reported to the coordinating office located within SKMCH\&RC. ${ }^{6}$ Both the paper-based forms and mobile data application, developed specifically for the Registry, are used. The variables on the form include; (1) demographic features: patient's name, age, birth date, gender, computerised national identity number, telephone number, address, and a question on whether the patient has come to Lahore for treatment only; (2) clinical features: the diagnosis date, primary tumour site, biopsy site, morphology, behaviour, grade, stage, metastasis, laterality, most valid basis of diagnosis, and procedure-related information (doctor's name and hospital); and (3) status at last follow-up, date and place of death. 5,6
Data are saved in the PCR database, which is an integral part of the Oracle-based Hospital Information System developed in-house by the information technology staff of SKMCH\&RC. This system also maintains the electronic health records of the patients.

Within the Registry, the incidence date is defined as the date of cytological/histological confirmation of a malignancy on a pathology report; the date of consultation at an outpatient clinic/walk-in clinic (without a clinical investigation or a tissue diagnosis); the date of clinical investigation(s) as imaging or tumour markers confirming the diagnosis; the date of admission to the hospital because of a malignancy; or the date of death, if no information is available other than the fact that the patient has died because of a malignancy. The proportion of cases microscopically verified (MV\%) is determined by including both the histologically confirmed cases and cases diagnosed on the basis of exfoliative cytology of the specimens.

Cancers are coded using the International Classification of Diseases, Clinical Modification, $10^{\text {th }}$ revision, and the International Classification of Diseases for Oncology, 3rd edition (ICD-O-3), 10,11 while multiple primaries are coded according to the multiple primary coding rules set by IARC.12 All cancers with behaviour codes $/ 2$ and $/ 3$ of ICD-O-3, including skin and bladder cancers, and tumours of the Central Nervous System ((CNS), brain and spinal cord) with behaviour codes $/ 0, / 1, / 2$, and $/ 3$, were included. Data were submitted to the IARC-CHECK programme to examine the data based on the following edits: gender versus site, age versus the date of birth, site versus morphology, site/histology versus age, histology versus behaviour, and histology versus the basis of diagnosis. A check for multiple primaries was done using the IARCcrgTools package.12 A check for duplicate records was done manually based on patient's name, telephone number, father's name, age, address, and primary site.

A retrospective review of the records within the PCR repository, for the period between 2010 and 2015, was carried out. The residents of Lahore diagnosed with cancer were selected. Annual ASIRs were computed using the Segi World Standard,13 applying the direct method of age-standardisation, and presented per 100,000 population, over a 6 -year period, by gender and tumour site/type. Sixteen 5-year age groups were created beginning from 0-4 to 70-74 years, followed by $75+$, and age-specific incidence rates computed. Data were analysed using Microsoft Excel V.2016 and SPSS V.19.

The Institutional Review Board (IRB) of SKMCH\&RC, registered with the OHRP as 'IRB00005898 - Shaukat Khanum Mem Cancer Hosp \& Rsch Centre IRB \#1 SKMCH \& RC', approved the study prior to its initiation (Reference No. 29-02-16-04). 


\section{RESULTS}

Over a 6-year period (2010-2015), a total of 33,028 new malignancies were recorded in Lahore among 32,977 patients, attributed to multiple primaries seen in 51 patients. Females accounted for $57.1 \%$ of the total patients. There were $5.8 \%$ children ( $0-14$ years), $2.2 \%$ young adults/adolescents (15-19 years), and $92 \%$ adults (20+ years). Approximately $43.6 \%$ of the patients were in

Table I: Annual age-specific incidence rates by 5-year age group and age-standardised incidence rates (ASIRs), per 100,000 females, Lahore, Pakistan, 2010-2015.

\begin{tabular}{|c|c|c|c|c|c|c|c|c|c|c|c|c|c|c|c|c|c|c|c|c|c|}
\hline Site & Cases & $0-$ & 5- & 10- & $15-$ & 20- & 25- & 30- & 35- & $40-$ & $45-$ & $50-$ & 55- & 60- & 65- & 70- & $75+$ & Crude & $\%$ & ASIR & $\begin{array}{l}\text { ICD-10 } \\
\text { code }\end{array}$ \\
\hline ip & 19 & 0.0 & 0.0 & 0.0 & 0.0 & 0.0 & 0.0 & 0.1 & 0.1 & 0.0 & 0.1 & 0.0 & 1.2 & 0.2 & 0.9 & 1.1 & 0.3 & 0.1 & 0.1 & 0.1 & $\mathrm{COO}$ \\
\hline Tongue & 274 & 0.0 & 0.0 & 0.0 & 0.0 & 0.1 & 0.1 & 0.5 & 1.1 & 1.5 & 4.4 & 3.8 & 6.5 & 8.3 & 6.4 & 5.3 & 5.8 & 0.9 & 1.5 & 1.7 & C01-C02 \\
\hline louth & 290 & 0.0 & 0.0 & 0.0 & 0.1 & 0.2 & 0.2 & 0.3 & 1.2 & 2.3 & 2.6 & 6.2 & 5.2 & 5.2 & 9.1 & 9.0 & 5.5 & 1.0 & 1.5 & 1.7 & C03-C06 \\
\hline Salivary glands & 93 & 0.0 & 0.0 & 0.1 & 0.1 & 0.1 & 0.4 & 0.4 & 0.7 & 0.3 & 0.8 & 1.3 & 0.8 & 2.0 & 2.1 & 2.3 & 1.0 & 0.3 & 0.5 & 0.5 & C07-C08 \\
\hline onsil & 4 & 0.0 & 0.0 & 0.0 & 0.0 & 0.0 & 0.0 & 0.0 & 0.1 & 0.1 & 0.0 & 0.1 & 0.2 & 0.0 & 0.0 & 0.0 & 0.0 & 0.0 & 0.0 & 0.0 & C10 \\
\hline asopharynx & 43 & 0.0 & 0.0 & 0.0 & 0.3 & 0.1 & 0.2 & 0.2 & 0.2 & 0.2 & 0.5 & 0.3 & 0.5 & 0.2 & 0.3 & 0.8 & 0.0 & 0.1 & 0.2 & 0.2 & C11 \\
\hline ypopharynx & 68 & 0.0 & 0.0 & 0.0 & 0.0 & 0.1 & 0.1 & 0.2 & 0.5 & 0.4 & 0.8 & 0.6 & 1.5 & 2.0 & 1.5 & 1.5 & 0.7 & 0.2 & 0.4 & 0.4 & C12-C13 \\
\hline harynx & 9 & 0.0 & 0.0 & 0.0 & 0.0 & 0.0 & 0.0 & 0.1 & 0.0 & 0.0 & 0.1 & 0.0 & 0.2 & 0.0 & 0.0 & 0.4 & 0.7 & 0.0 & 0.0 & 0.0 & C14 \\
\hline Desophagus & 213 & 0.0 & 0.0 & 0.0 & 0.0 & 0.1 & 0.3 & 0.7 & 1.1 & 1.2 & 3.8 & 2.2 & 2.8 & 5.5 & 5.8 & 4.9 & 4.8 & 0.7 & 1.1 & 1.3 & C15 \\
\hline Stomach & 217 & 0.0 & 0.0 & 0.0 & 0.0 & 0.2 & 0.2 & 0.9 & 1.1 & 1.9 & 3.2 & 2.9 & 4.3 & 3.3 & 7.3 & 2.3 & 3.4 & 0.7 & 1.2 & 1.2 & C16 \\
\hline mall intestine & 53 & 0.0 & 0.0 & 0.0 & 0.0 & 0.1 & 0.0 & 0.1 & 0.1 & 0.3 & 0.9 & 0.1 & 0.5 & 1.8 & 2.1 & 1.9 & 2.1 & 0.2 & 0.3 & 0.3 & C17 \\
\hline Colon & 328 & 0.0 & 0.0 & 0.1 & 0.3 & 0.3 & 0.8 & 1.0 & 1.5 & 2.0 & 3.3 & 3.6 & 6.8 & 5.5 & 8.8 & 7.9 & 8.9 & 1.1 & 1.7 & 1.9 & C18 \\
\hline Rectum & 290 & 0.0 & 0.0 & 0.1 & 0.4 & 0.9 & 0.9 & 1.0 & 1.3 & 1.7 & 2.7 & 2.7 & 3.5 & 5.7 & 7.3 & 6.4 & 5.8 & 1.0 & 1.5 & 1.6 & C19-C20 \\
\hline Anus & 54 & 0.0 & 0.0 & 0.0 & 0.0 & 0.2 & 0.1 & 0.3 & 0.1 & 0.7 & 0.5 & 0.5 & 0.3 & 0.9 & 1.2 & 1.5 & 0.7 & 0.2 & 0.3 & 0.3 & $\mathrm{C} 21$ \\
\hline iver & 288 & 0.2 & 0.0 & 0.0 & 0.1 & 0.2 & 0.2 & 0.3 & 0.3 & 0.9 & 3.1 & 4.5 & 8.7 & 9.6 & 11.5 & 5.3 & 3.8 & 1.0 & 1.5 & 1.8 & $\mathrm{C} 22$ \\
\hline Gall bladder, etc. ${ }^{*}$ & 276 & 0.0 & 0.0 & 0.0 & 0.0 & 0.0 & 0.0 & 0.5 & 0.5 & 1.4 & 2.7 & 4.2 & 6.3 & 8.3 & 13.6 & 8.6 & 6.2 & 0.9 & 1.5 & 1.8 & C23-C24 \\
\hline Dancreas & 60 & 0.0 & 0.0 & 0.0 & 0.1 & 0.0 & 0.1 & 0.1 & 0.3 & 0.2 & 0.8 & 0.8 & 1.0 & 1.3 & 3.6 & 1.5 & 0.7 & 0.2 & 0.3 & 0.4 & $\mathrm{C} 25$ \\
\hline Jose, sinuses & 45 & 0.0 & 0.0 & 0.0 & 0.1 & 0.0 & 0.0 & 0.2 & 0.2 & 0.0 & 0.7 & 0.5 & 0.8 & 1.7 & 1.5 & 0.4 & 1.0 & 0.2 & 0.2 & 0.3 & C30-C31 \\
\hline $\operatorname{arynx}$ & 50 & 0.0 & 0.0 & 0.0 & 0.0 & 0.0 & 0.0 & 0.2 & 0.1 & 0.4 & 0.4 & 1.0 & 1.5 & 0.9 & 0.9 & 0.8 & 2.4 & 0.2 & 0.3 & 0.3 & C32 \\
\hline Irachea, bronchus, lung & 225 & 0.0 & 0.0 & 0.1 & 0.1 & 0.1 & 0.1 & 0.7 & 0.8 & 1.2 & 1.2 & 3.4 & 6.2 & 5.7 & 7.6 & 6.8 & 4.5 & 0.8 & 1.2 & 1.4 & C33-C34 \\
\hline Dther thoracic organs & 28 & 0.0 & 0.0 & 0.1 & 0.0 & 0.0 & 0.0 & 0.0 & 0.1 & 0.1 & 0.5 & 0.2 & 0.2 & 0.7 & 0.6 & 0.8 & 1.4 & 0.1 & 0.1 & 0.2 & C37-C39 \\
\hline Bone & 184 & 0.1 & 0.4 & 0.9 & 1.5 & 0.8 & 0.3 & 0.3 & 0.8 & 0.4 & 0.8 & 0.4 & 0.7 & 0.6 & 0.6 & 1.1 & 1.0 & 0.6 & 1.0 & 0.6 & C40-C41 \\
\hline telanoma of skin & 38 & 0.0 & 0.0 & 0.0 & 0.0 & 0.0 & 0.1 & 0.2 & 0.1 & 0.1 & 0.3 & 0.2 & 0.5 & 0.0 & 0.6 & 0.4 & 0.3 & 0.1 & 0.1 & 0.2 & $\mathrm{C} 43$ \\
\hline ther skin & 402 & 0.1 & 0.0 & 0.1 & 0.1 & 0.2 & 0.3 & 0.6 & 1.5 & 2.6 & 2.7 & 4.9 & 6.5 & 11.4 & 14.6 & 18.0 & 19.3 & 1.4 & 2.2 & 2.6 & C44 \\
\hline Mesothelioma & 5 & 0.0 & 0.0 & 0.0 & 0.0 & 0.0 & 0.0 & 0.0 & 0.0 & 0.0 & 0.0 & 0.1 & 0.0 & 0.4 & 0.0 & 0.4 & 0.0 & 0.0 & 0.0 & 0.0 & C45 \\
\hline Connective and sof & 232 & 0.4 & 0.2 & 0.3 & 0.6 & 0.5 & 0.7 & 0.6 & 1.5 & 1.5 & 1.5 & 2.4 & 2.7 & 2.8 & 3.9 & 1.9 & 2.1 & 0.8 & 1.2 & 1.1 & C47,C49 \\
\hline Breast & 8340 & 0.0 & 0.0 & 0.1 & 0.1 & 2.8 & 14.2 & 29.8 & 56.3 & 81.1 & 119.8 & 120.7 & 161.0 & 152.9 & 162.0 & 117.8 & 99.4 & 28.3 & 44.3 & 46.4 & C50 \\
\hline Vulva & 34 & 0.0 & 0.0 & 0.0 & 0.0 & 0.0 & 0.0 & 0.1 & 0.2 & 0.1 & 0.2 & 0.4 & 0.7 & 1.3 & 1.2 & 0.8 & 1.7 & 0.1 & 0.2 & 0.2 & C51 \\
\hline Vagina & 38 & 0.0 & 0.0 & 0.0 & 0.0 & 0.0 & 0.1 & 0.0 & 0.3 & 0.2 & 0.7 & 0.6 & 0.8 & 0.6 & 0.6 & 0.8 & 1.0 & 0.1 & 0.2 & 0.2 & C52 \\
\hline Cervix uteri & 491 & 0.0 & 0.0 & 0.0 & 0.0 & 0.2 & 0.4 & 1.3 & 3.3 & 5.3 & 8.5 & 7.0 & 9.3 & 8.7 & 10.0 & 6.0 & 5.5 & 1.7 & 2.6 & 2.8 & C53 \\
\hline Corpus uteri & 636 & 0.0 & 0.0 & 0.0 & 0.0 & 0.0 & 0.3 & 0.5 & 1.5 & 2.1 & 6.9 & 10.6 & 20.0 & 21.2 & 22.4 & 21.8 & 8.3 & 2.2 & 3.4 & 4.1 & C54 \\
\hline Jterus, unspe & 176 & 0.0 & 0.0 & 0.0 & 0.0 & 0.0 & 0.1 & 0.1 & 0.9 & 1.2 & 2.5 & 3.4 & 3.8 & 5.9 & 3.9 & 4.5 & 0.7 & 0.6 & 0.9 & 1.1 & C55 \\
\hline Dvary & 877 & 0.0 & 0.1 & 0.6 & 0.6 & 1.4 & 2.3 & 2.4 & 4.1 & 7.3 & 11.9 & 11.7 & 16.3 & 15.3 & 16.7 & 10.1 & 6.9 & 3.0 & 4.7 & 4.7 & C56 \\
\hline ther fema & 51 & 0.0 & 0.0 & 0.0 & 0.0 & 0.0 & 0.0 & 0.1 & 0.3 & 0.3 & 0.8 & 1.0 & 0.8 & 1.7 & 1.5 & 0.8 & 0.0 & 0.2 & 0.3 & 0.3 & C57 \\
\hline Placenta & 15 & 0.0 & 0.0 & 0.0 & 0.0 & 0.1 & 0.1 & 0.3 & 0.1 & 0.1 & 0.1 & 0.0 & 0.0 & 0.0 & 0.0 & 0.0 & 0.0 & 0.1 & 0.1 & 0.1 & C58 \\
\hline Sidney & 210 & 0.5 & 0.2 & 0.1 & 0.0 & 0.1 & 0.0 & 0.5 & 1.1 & 1.3 & 1.9 & 3.1 & 5.2 & 3.3 & 3.3 & 4.1 & 2.8 & 0.7 & 1.1 & 1.1 & C64 \\
\hline Renal pelvis & 1 & 0.0 & 0.0 & 0.0 & 0.0 & 0.0 & 0.0 & 0.0 & 0.0 & 0.0 & 0.0 & 0.1 & 0.0 & 0.0 & 0.0 & 0.0 & 0.0 & 0.0 & 0.0 & 0.0 & $\mathrm{C} 65$ \\
\hline Jreter & 1 & 0.0 & 0.0 & 0.0 & 0.0 & 0.0 & 0.0 & 0.0 & 0.0 & 0.0 & 0.0 & 0.0 & 0.0 & 0.0 & 0.0 & 0.4 & 0.0 & 0.0 & 0.0 & 0.0 & C66 \\
\hline Bladder & 226 & 0.1 & 0.0 & 0.0 & 0.1 & 0.1 & 0.2 & 0.2 & 1.0 & 1.0 & 1.8 & 2.4 & 4.8 & 5.2 & 8.8 & 9.0 & 10.0 & 0.8 & 1.2 & 1.4 & C67 \\
\hline Other urinary organs & 3 & 0.0 & 0.0 & 0.0 & 0.0 & 0.0 & 0.0 & 0.0 & 0.0 & 0.0 & 0.1 & 0.0 & 0.2 & 0.0 & 0.0 & 0.4 & 0.0 & 0.0 & 0.0 & 0.0 & C68 \\
\hline Eye & 92 & 1.1 & 0.3 & 0.1 & 0.0 & 0.0 & 0.0 & 0.1 & 0.1 & 0.4 & 0.4 & 0.1 & 0.2 & 0.4 & 2.4 & 0.8 & 1.7 & 0.3 & 0.5 & 0.4 & C69 \\
\hline Brain, nervous system & 559 & 0.4 & 0.9 & 1.0 & 1.2 & 1.1 & 2.0 & 2.2 & 2.7 & 3.7 & 4.6 & 6.0 & 5.8 & 6.1 & 6.1 & 4.5 & 2.8 & 1.9 & 3.0 & 2.5 & C70-C72 \\
\hline Thyroid & 440 & 0.0 & 0.0 & 0.1 & 0.4 & 1.8 & 2.1 & 2.6 & 3.3 & 3.5 & 4.1 & 4.8 & 3.2 & 4.4 & 3.3 & 7.5 & 2.1 & 1.5 & 2.3 & 2.0 & $\mathrm{C} 73$ \\
\hline Adrenal & 9 & 0.0 & 0.0 & 0.0 & 0.0 & 0.1 & 0.0 & 0.0 & 0.1 & 0.1 & 0.0 & 0.0 & 0.0 & 0.4 & 0.3 & 0.0 & 0.0 & 0.0 & 0.0 & 0.0 & C74 \\
\hline Other endocrine & 1 & 0.0 & 0.0 & 0.0 & 0.0 & 0.0 & 0.0 & 0.0 & 0.0 & 0.0 & 0.0 & 0.0 & 0.0 & 0.0 & 0.0 & 0.0 & 0.0 & 0.0 & 0.0 & 0.0 & C75 \\
\hline Todgkin lymphoma & 168 & 0.2 & 0.4 & 0.3 & 0.6 & 0.9 & 0.8 & 0.5 & 0.6 & 0.6 & 1.0 & 0.7 & 1.5 & 1.1 & 1.2 & 2.3 & 0.0 & 0.6 & 0.9 & 0.7 & C81 \\
\hline Non-Hodgkin lymphoma & 590 & 0.3 & 0.5 & 0.3 & 0.5 & 0.8 & 0.8 & 1.4 & 2.0 & 3.1 & 5.4 & 8.8 & 12.0 & 11.2 & 11.2 & 14.6 & 12.7 & 2.0 & 3.1 & 3.3 & $\begin{array}{c}\text { C82- } \\
\text { C86, } \\
88.4, \\
\text { C96 }\end{array}$ \\
\hline Multiple myeloma & 76 & 0.0 & 0.0 & 0.0 & 0.0 & 0.0 & 0.0 & 0.0 & 0.2 & 0.5 & 1.1 & 1.3 & 1.2 & 2.9 & 3.0 & 2.6 & 0.7 & 0.3 & 0.4 & 0.5 & $\mathrm{C} 90$ \\
\hline Lymphoid leukaemia & 240 & 2.1 & 1.6 & 1.2 & 0.4 & 0.2 & 0.1 & 0.2 & 0.2 & 0.2 & 0.2 & 0.1 & 0.7 & 0.6 & 0.6 & 1.1 & 0.7 & 0.8 & 1.3 & 0.7 & C91 \\
\hline Myeloid leukaemia & 152 & 0.2 & 0.1 & 0.2 & 0.3 & 0.6 & 0.9 & 1.0 & 0.9 & 0.4 & 0.9 & 0.8 & 2.0 & 1.8 & 0.6 & 1.1 & 0.0 & 0.5 & 0.8 & 0.6 & C92-94 \\
\hline Leukaemia unspecified & 76 & 0.5 & 0.4 & 0.2 & 0.2 & 0.1 & 0.2 & 0.2 & 0.1 & 0.3 & 0.2 & 0.3 & 0.3 & 0.0 & 0.6 & 0.4 & 0.3 & 0.3 & 0.4 & 0.3 & $\mathrm{C} 95$ \\
\hline Other and unspecified & 1190 & 0.5 & 0.2 & 0.2 & 0.4 & 1.3 & 1.8 & 2.5 & 3.8 & 5.5 & 13.8 & 15.1 & 26.1 & 25.8 & 36.4 & 30.8 & 31.3 & 4.0 & 6.3 & 7.1 & $\begin{array}{l}\text { O\&U } \\
\text { Other } \\
\text { benign }\end{array}$ \\
\hline Benign $\mathrm{CNS}^{\star *}$ & 367 & 0.1 & 0.1 & 0.3 & 0.5 & 0.7 & 1.6 & 2.0 & 3.2 & 3.2 & 3.9 & 4.4 & 2.5 & 3.7 & 3.9 & 2.6 & 0.7 & 1.2 & 1.9 & 1.7 & CNS \\
\hline All sites & 18847 & 6.9 & 5.7 & 6.3 & 9.0 & 16.7 & 33.6 & 57.0 & 101.5 & 142.7 & 229.0 & 250.6 & 347.5 & 358.3 & 412.2 & 335.4 & 271.8 & 64.0 & 100.0 & $\mid 103.9$ & C00-C96 \\
\hline
\end{tabular}


the 45-64 year age group. The mean age at presentation was $48.7 \pm 18.4$ years with median $=50$ years, and mode $=$ 60 years. Nearly $96.2 \%$ of the cancers were microscopically verified (histology of primary tumour in $83 \%$, histology of a metastasis in $7.9 \%$, and cytology in $5.3 \%$ of the cases), $2.6 \%$ were clinically diagnosed, $1.2 \%$ through clinical investigations, and none by death certificates only. The $\mathrm{MV} \%$ was $88.3 \%-100 \%$ among females and $76.2 \%-100 \%$ in males, except for liver cancer in either gender where it was $50 \%$.

The ASIR was 103.9 in females and 66.5 in males, per 100,000 population. The ASIR was higher in males than

Table II: Annual age-specific incidence rates by 5-year age group and age-standardised incidence rates (ASIRs), per 100,000 males, Lahore, Pakistan, 2010-2015.

\begin{tabular}{|c|c|c|c|c|c|c|c|c|c|c|c|c|c|c|c|c|c|c|c|c|c|}
\hline Site & Total & $0-$ & 5- & 10- & $15-$ & $20-$ & 25- & 30- & $35-$ & 40- & 45- & $50-$ & 55- & $60-$ & 65- & 70- & $75+$ & Crude & $\%$ & ASIR & $\begin{array}{c}\text { ICD-10 } \\
\text { code }\end{array}$ \\
\hline Lip & 33 & 0.0 & 0.0 & 0.0 & 0.0 & 0.0 & 0.0 & 0.1 & 0.2 & 0.1 & 0.1 & 0.4 & 0.5 & 0.9 & 0.7 & 0.6 & 1.1 & 0.1 & 0.2 & 0.2 & $\mathrm{C} 00$ \\
\hline Tongue & 355 & 0.0 & 0.0 & 0.0 & 0.0 & 0.2 & 0.4 & 0.5 & 1.6 & 3.0 & 3.9 & 3.9 & 5.0 & 6.4 & 6.1 & 9.0 & 4.1 & 1.1 & 2.5 & 1.7 & C01-C02 \\
\hline Mouth & 481 & 0.0 & 0.0 & 0.0 & 0.0 & 0.1 & 0.3 & 1.1 & 1.7 & 3.5 & 6.2 & 5.8 & 9.6 & 9.4 & 8.6 & 7.0 & 4.1 & 1.5 & 3.4 & 2.3 & C03-C06 \\
\hline Salivary glands & 117 & 0.0 & 0.0 & 0.0 & 0.1 & 0.1 & 0.3 & 0.3 & 0.5 & 0.9 & 0.7 & 1.2 & 1.5 & 2.1 & 1.5 & 2.3 & 2.7 & 0.4 & 0.8 & 0.5 & C07-C08 \\
\hline Tonsil & 6 & 0.0 & 0.0 & 0.0 & 0.0 & 0.0 & 0.0 & 0.0 & 0.0 & 0.0 & 0.2 & 0.0 & 0.3 & 0.1 & 0.0 & 0.3 & 0.0 & 0.0 & 0.0 & 0.0 & C10 \\
\hline Vasopharynx & 57 & 0.0 & 0.0 & 0.1 & 0.2 & 0.1 & 0.0 & 0.2 & 0.3 & 0.3 & 0.5 & 0.5 & 0.9 & 0.6 & 0.5 & 0.3 & 0.3 & 0.2 & 0.4 & 0.2 & C11 \\
\hline Hypopharynx & 48 & 0.0 & 0.0 & 0.0 & 0.0 & 0.0 & 0.2 & 0.0 & 0.1 & 0.4 & 0.2 & 0.5 & 0.4 & 1.2 & 1.0 & 1.1 & 1.6 & 0.1 & 0.3 & 0.2 & C12-C13 \\
\hline Pharynx & 14 & 0.0 & 0.0 & 0.0 & 0.0 & 0.0 & 0.0 & 0.1 & 0.2 & 0.1 & 0.2 & 0.3 & 0.1 & 0.1 & 0.0 & 0.0 & 0.0 & 0.0 & 0.1 & 0.1 & C14 \\
\hline Oesophagus & 295 & 0.0 & 0.0 & 0.0 & 0.1 & 0.1 & 0.3 & 0.4 & 0.7 & 1.0 & 2.5 & 3.0 & 5.4 & 5.8 & 9.1 & 8.7 & 7.9 & 0.9 & 2.1 & 1.5 & C15 \\
\hline Stomach & 332 & 0.0 & 0.0 & 0.0 & 0.0 & 0.1 & 0.6 & 0.8 & 1.2 & 1.5 & 4.1 & 3.2 & 6.1 & 5.2 & 9.3 & 5.9 & 5.2 & 1.0 & 2.3 & 1.6 & C16 \\
\hline Small intestine & 62 & 0.0 & 0.0 & 0.0 & 0.0 & 0.1 & 0.1 & 0.0 & 0.2 & 0.2 & 0.4 & 0.7 & 1.1 & 0.7 & 1.0 & 2.8 & 1.9 & 0.2 & 0.4 & 0.3 & C17 \\
\hline Colon & 486 & 0.0 & 0.0 & 0.1 & 0.2 & 0.6 & 0.6 & 1.1 & 1.8 & 2.5 & 4.7 & 5.2 & 6.2 & 9.7 & 12.8 & 9.8 & 6.5 & 1.5 & 3.4 & 2.3 & C18 \\
\hline Rectum & 396 & 0.0 & 0.0 & 0.0 & 0.4 & 0.6 & 0.8 & 1.4 & 1.4 & 1.2 & 2.9 & 3.6 & 4.6 & 7.8 & 11.3 & 6.8 & 7.3 & 1.2 & 2.8 & 1.9 & C19-C20 \\
\hline Anus & 78 & 0.0 & 0.0 & 0.0 & 0.1 & 0.1 & 0.1 & 0.4 & 0.1 & 0.4 & 0.7 & 0.8 & 0.8 & 1.5 & 2.5 & 0.8 & 1.1 & 0.2 & 0.6 & 0.4 & $\mathrm{C} 21$ \\
\hline iver & 557 & 0.3 & 0.0 & 0.0 & 0.0 & 0.1 & 0.1 & 0.1 & 0.7 & 1.8 & 3.7 & 7.3 & 12.4 & 12.5 & 22.1 & 13.8 & 12.0 & 1.7 & 3.9 & 3.0 & $\mathrm{C} 22$ \\
\hline Gall bladder, etc. ${ }^{*}$ & 182 & 0.0 & 0.0 & 0.0 & 0.0 & 0.0 & 0.0 & 0.2 & 0.3 & 1.1 & 0.9 & 2.2 & 3.8 & 4.5 & 6.1 & 3.7 & 4.9 & 0.6 & 1.3 & 1.0 & C23-C24 \\
\hline Pancreas & 101 & 0.0 & 0.0 & 0.0 & 0.0 & 0.0 & 0.0 & 0.1 & 0.0 & 0.5 & 0.8 & 1.0 & 2.0 & 2.7 & 3.9 & 3.7 & 1.6 & 0.3 & 0.7 & 0.5 & $\mathrm{C} 25$ \\
\hline Nose, sinuses & 71 & 0.0 & 0.0 & 0.0 & 0.1 & 0.1 & 0.2 & 0.2 & 0.3 & 0.4 & 0.4 & 0.3 & 0.9 & 1.5 & 0.7 & 3.1 & 0.8 & 0.2 & 0.5 & 0.3 & C30-C31 \\
\hline Larynx & 408 & 0.0 & 0.0 & 0.0 & 0.0 & 0.2 & 0.0 & 0.3 & 0.7 & 1.4 & 3.6 & 4.9 & 8.6 & 10.9 & 11.5 & 12.1 & 8.4 & 1.2 & 2.9 & 2.2 & $\mathrm{C} 32$ \\
\hline Trachea, bronchus, lung & 844 & 0.0 & 0.0 & 0.0 & 0.0 & 0.1 & 0.1 & 0.5 & 1.4 & 1.8 & 4.7 & 7.3 & 13.7 & 19.9 & 35.1 & 36.0 & 33.1 & 2.6 & 6.0 & 4.7 & C33-C34 \\
\hline Other thoracic organs & 70 & 0.0 & 0.0 & 0.1 & 0.1 & 0.0 & 0.1 & 0.3 & 0.1 & 0.5 & 0.4 & 1.2 & 0.1 & 1.3 & 0.2 & 1.1 & 2.2 & 0.2 & 0.5 & 0.3 & C37-C39 \\
\hline Bone & 286 & 0.2 & 0.4 & 1.1 & 2.3 & 1.4 & 0.5 & 0.7 & 0.4 & 0.7 & 0.7 & 0.5 & 0.5 & 1.9 & 0.5 & 0.8 & 0.5 & 0.9 & 2.0 & 0.8 & C40-C41 \\
\hline Melanoma of skin & 44 & 0.0 & 0.0 & 0.0 & 0.0 & 0.0 & 0.0 & 0.0 & 0.1 & 0.1 & 0.0 & 0.2 & 0.7 & 0.7 & 0.2 & 0.0 & 1.1 & 0.1 & 0.2 & 0.2 & $\mathrm{C} 43$ \\
\hline Other skin & 589 & 0.1 & 0.1 & 0.1 & 0.1 & 0.3 & 0.6 & 1.5 & 1.8 & 2.1 & 3.1 & 5.4 & 9.4 & 11.0 & 17.7 & 15.8 & 25.5 & 1.9 & 4.3 & 3.0 & C44 \\
\hline Mesothelioma & 6 & 0.0 & 0.0 & 0.0 & 0.0 & 0.0 & 0.0 & 0.0 & 0.1 & 0.1 & 0.0 & 0.0 & 0.0 & 0.0 & 0.0 & 0.3 & 0.5 & 0.0 & 0.0 & 0.0 & $\mathrm{C} 45$ \\
\hline Connective and soft tissue & 276 & 0.5 & 0.4 & 0.2 & 0.6 & 0.8 & 0.8 & 0.7 & 0.9 & 0.7 & 2.0 & 1.1 & 3.2 & 2.7 & 4.4 & 2.8 & 1.9 & 0.8 & 1.9 & 1.1 & C47,C49 \\
\hline Breast & 166 & 0.0 & 0.0 & 0.0 & 0.0 & 0.0 & 0.1 & 0.1 & 0.4 & 0.9 & 2.3 & 1.3 & 2.9 & 3.1 & 6.4 & 4.5 & 2.2 & 0.5 & 1.2 & 0.9 & C50 \\
\hline Penis & 6 & 0.0 & 0.0 & 0.0 & 0.0 & 0.0 & 0.0 & 0.0 & 0.1 & 0.1 & 0.0 & 0.0 & 0.3 & 0.1 & 0.0 & 0.0 & 0.0 & 0.0 & 0.0 & 0.0 & $\mathrm{C} 60$ \\
\hline Prostate & 1174 & 0.0 & 0.0 & 0.0 & 0.0 & 0.1 & 0.0 & 0.0 & 0.1 & 0.2 & 1.5 & 4.5 & 13.3 & 28.5 & 53.0 & 68.9 & 93.4 & 3.6 & 8.3 & 6.8 & C61 \\
\hline Testis & 196 & 0.3 & 0.0 & 0.0 & 0.5 & 1.0 & 1.5 & 1.3 & 1.3 & 0.7 & 0.6 & 0.4 & 0.4 & 0.3 & 1.0 & 0.6 & 0.5 & 0.6 & 1.4 & 0.6 & C62 \\
\hline Other male genital org & 5 & 0.0 & 0.0 & 0.0 & 0.0 & 0.0 & 0.0 & 0.0 & 0.0 & 0.0 & 0.0 & 0.0 & 0.0 & 0.0 & 0.5 & 0.3 & 0.0 & 0.0 & 0.0 & 0.0 & C63 \\
\hline Kidney & 352 & 0.8 & 0.2 & 0.0 & 0.1 & 0.1 & 0.2 & 0.6 & 0.9 & 2.2 & 2.6 & 3.2 & 4.2 & 7.0 & 9.1 & 7.6 & 4.9 & 1.1 & 2.5 & 1.7 & C64 \\
\hline Renal pelvis & 4 & 0.0 & 0.0 & 0.0 & 0.0 & 0.0 & 0.0 & 0.0 & 0.0 & 0.0 & 0.1 & 0.0 & 0.3 & 0.0 & 0.0 & 0.0 & 0.3 & 0.0 & 0.0 & 0.0 & C65 \\
\hline Ureter & 1 & 0.0 & 0.0 & 0.0 & 0.0 & 0.0 & 0.0 & 0.0 & 0.0 & 0.0 & 0.0 & 0.0 & 0.1 & 0.0 & 0.0 & 0.0 & 0.0 & 0.0 & 0.0 & 0.0 & C66 \\
\hline Bladder & 957 & 0.0 & 0.0 & 0.0 & 0.0 & 0.1 & 0.3 & 0.6 & 1.6 & 2.3 & 5.7 & 7.5 & 17.4 & 25.8 & 30.9 & 35.2 & 40.7 & 2.9 & 6.7 & 5.2 & C67 \\
\hline Other urinary organs & 3 & 0.0 & 0.0 & 0.0 & 0.0 & 0.0 & 0.0 & 0.0 & 0.0 & 0.0 & 0.0 & 0.0 & 0.1 & 0.0 & 0.0 & 0.0 & 0.3 & 0.0 & 0.0 & 0.0 & C68 \\
\hline Eye & 132 & 1.2 & 0.4 & 0.1 & 0.1 & 0.1 & 0.1 & 0.1 & 0.1 & 0.2 & 0.6 & 0.5 & 0.3 & 1.8 & 2.0 & 1.4 & 1.4 & 0.4 & 0.9 & 0.5 & C69 \\
\hline Brain, nervous system & 952 & 0.7 & 1.2 & 0.9 & 1.6 & 1.9 & 3.2 & 4.1 & 3.8 & 4.2 & 6.9 & 7.3 & 9.9 & 10.8 & 10.8 & 6.8 & 4.1 & 2.9 & 6.7 & 3.7 & C70-C72 \\
\hline Thyroid & 181 & 0.0 & 0.0 & 0.0 & 0.2 & 0.3 & 0.7 & 0.9 & 0.8 & 0.9 & 1.6 & 2.1 & 2.2 & 1.2 & 2.5 & 2.5 & 1.1 & 0.6 & 1.3 & 0.7 & $\mathrm{C} 73$ \\
\hline Adrenal & 10 & 0.1 & 0.0 & 0.0 & 0.0 & 0.1 & 0.0 & 0.0 & 0.0 & 0.0 & 0.1 & 0.0 & 0.1 & 0.0 & 0.2 & 0.3 & 0.0 & 0.0 & 0.1 & 0.0 & $\mathrm{C} 74$ \\
\hline Other endocrine & 5 & 0.0 & 0.0 & 0.0 & 0.0 & 0.0 & 0.0 & 0.0 & 0.0 & 0.1 & 0.0 & 0.0 & 0.0 & 0.0 & 0.2 & 0.0 & 0.0 & 0.0 & 0.0 & 0.0 & $\mathrm{C} 75$ \\
\hline Hodgkin lymphoma & 394 & 0.7 & 1.5 & 0.8 & 1.0 & 1.1 & 1.3 & 1.1 & 1.2 & 1.3 & 2.0 & 1.5 & 2.4 & 1.5 & 2.9 & 2.8 & 0.8 & 1.2 & 2.8 & 1.3 & $\mathrm{C} 81$ \\
\hline Non-Hodgkin lymphoma & 1047 & 0.5 & 1.2 & 1.1 & 1.4 & 1.6 & 1.8 & 2.1 & 3.2 & 4.3 & 6.2 & 9.3 & 12.9 & 18.1 & 17.4 & 17.7 & 16.8 & 3.2 & 7.4 & 4.6 & $\begin{array}{c}\text { C82- } \\
\text { C86,C88, } \\
\text { C96 }\end{array}$ \\
\hline Immunoproliferative dis. & 1 & 0.0 & 0.0 & 0.0 & 0.0 & 0.0 & 0.0 & 0.0 & 0.0 & 0.0 & 0.0 & 0.0 & 0.1 & 0.0 & 0.0 & 0.0 & 0.0 & 0.0 & 0.0 & 0.0 & C88 \\
\hline Multiple myeloma & 97 & 0.0 & 0.0 & 0.0 & 0.0 & 0.0 & 0.0 & 0.1 & 0.2 & 0.4 & 1.0 & 1.3 & 2.5 & 2.4 & 1.2 & 2.3 & 2.7 & 0.3 & 0.7 & 0.5 & $\mathrm{C} 90$ \\
\hline Lymphoid leukaemia & 418 & 3.0 & 2.4 & 2.0 & 0.7 & 0.4 & 0.3 & 0.3 & 0.1 & 0.5 & 0.9 & 1.0 & 1.3 & 0.7 & 1.7 & 1.4 & 1.1 & 1.3 & 2.9 & 1.2 & C91 \\
\hline Myeloid leukaemia & 230 & 0.3 & 0.3 & 0.4 & 0.4 & 0.6 & 0.8 & 1.0 & 1.2 & 1.4 & 1.4 & 0.8 & 2.2 & 2.1 & 1.7 & 0.3 & 0.5 & 0.7 & 1.6 & 0.8 & C92-94 \\
\hline Leukaemia unspecified & 118 & 0.7 & 0.5 & 0.7 & 0.1 & 0.3 & 0.1 & 0.1 & 0.2 & 0.2 & 0.2 & 0.1 & 0.4 & 0.3 & 0.0 & 0.3 & 0.0 & 0.4 & 0.8 & 0.3 & C95 \\
\hline Myelodysplastic synd. & 1 & 0.0 & 0.0 & 0.0 & 0.0 & 0.0 & 0.0 & 0.0 & 0.0 & 0.1 & 0.0 & 0.0 & 0.0 & 0.0 & 0.0 & 0.0 & 0.0 & 0.0 & 0.0 & 0.0 & MDS \\
\hline Myeloproliferative dis. & 4 & 0.0 & 0.0 & 0.0 & 0.0 & 0.0 & 0.0 & 0.0 & 0.0 & 0.1 & 0.0 & 0.1 & 0.1 & 0.0 & 0.0 & 0.0 & 0.3 & 0.0 & 0.0 & 0.0 & MPD \\
\hline Other and unspecified & 1158 & 0.4 & 0.2 & 0.4 & 0.9 & 0.6 & 1.7 & 2.1 & 2.9 & 4.3 & 6.9 & 11.4 & 18.0 & 22.8 & 29.9 & 29.0 & 32.3 & 3.5 & 8.2 & 5.7 & O\&U \\
\hline Benign $\mathrm{CNS}^{\star \star}$ & 375 & 0.2 & 0.3 & 0.2 & 0.5 & 0.7 & 1.6 & 1.5 & 2.6 & 2.4 & 3.6 & 2.9 & 2.4 & 3.0 & 1.7 & 3.4 & 0.8 & 1.1 & 2.6 & 1.4 & $\begin{array}{c}\text { Other } \\
\text { benign } \\
\text { CNS }\end{array}$ \\
\hline All sites & 14181 & 9.9 & 9.5 & 8.6 & 12.0 & 14.2 & 19.9 & 27.1 & 37.3 & 53.0 & 91.8 & 115.9 & 191.9 & 251.0 & 339.9 & 333.8 & 340.6 & 43.3 & 100.0 & 66.5 & Total \\
\hline
\end{tabular}

${ }^{*}$ Gall bladder, etc = Includes the extrahepatic bile ducts; $\quad{ }^{* *}$ CNS = Central Nervous System. 
Table III: Global comparison of the age-standardised incidence rates, per 100,000 population, by cancer site/type and gender.

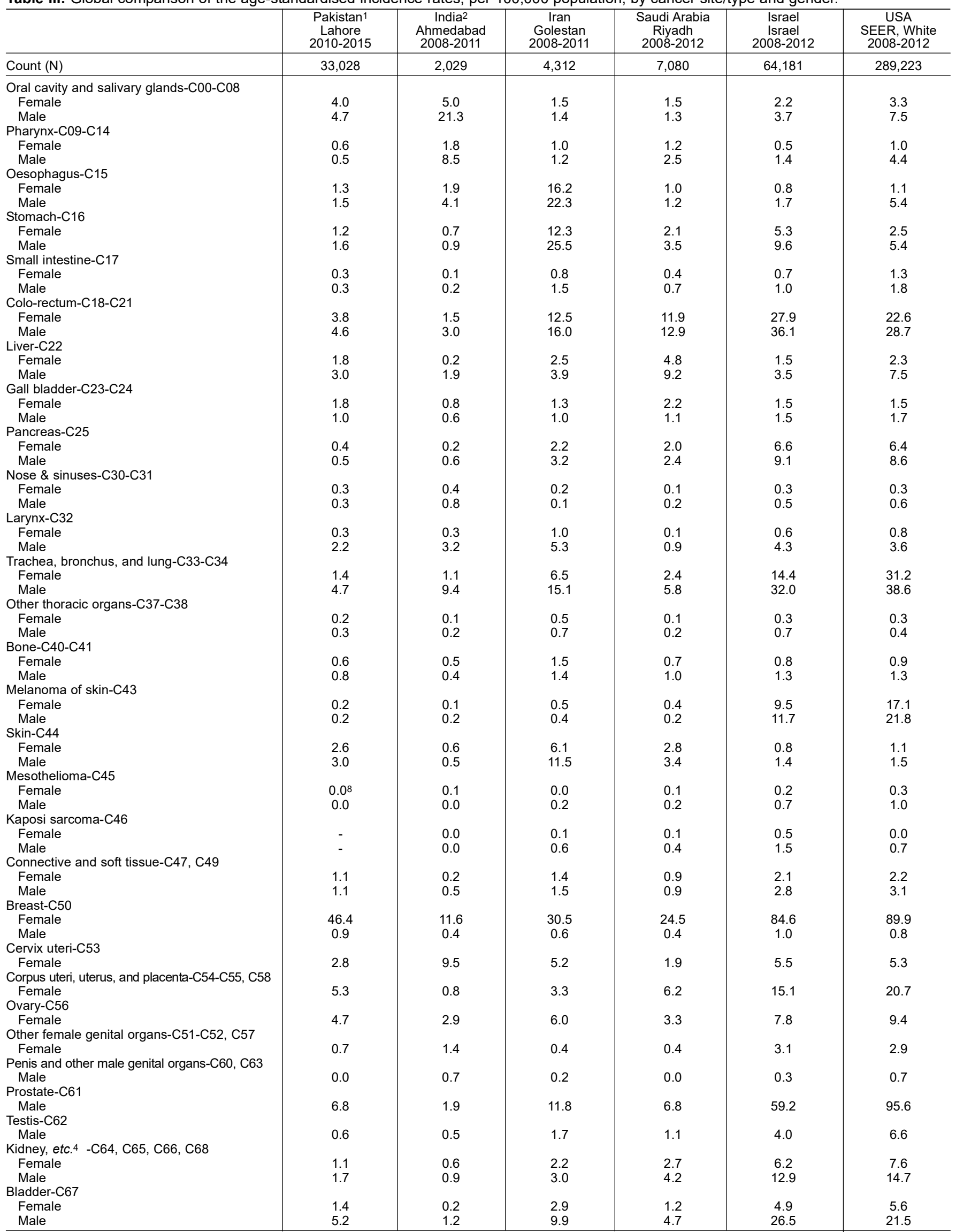

Continues... 
Table III continues....

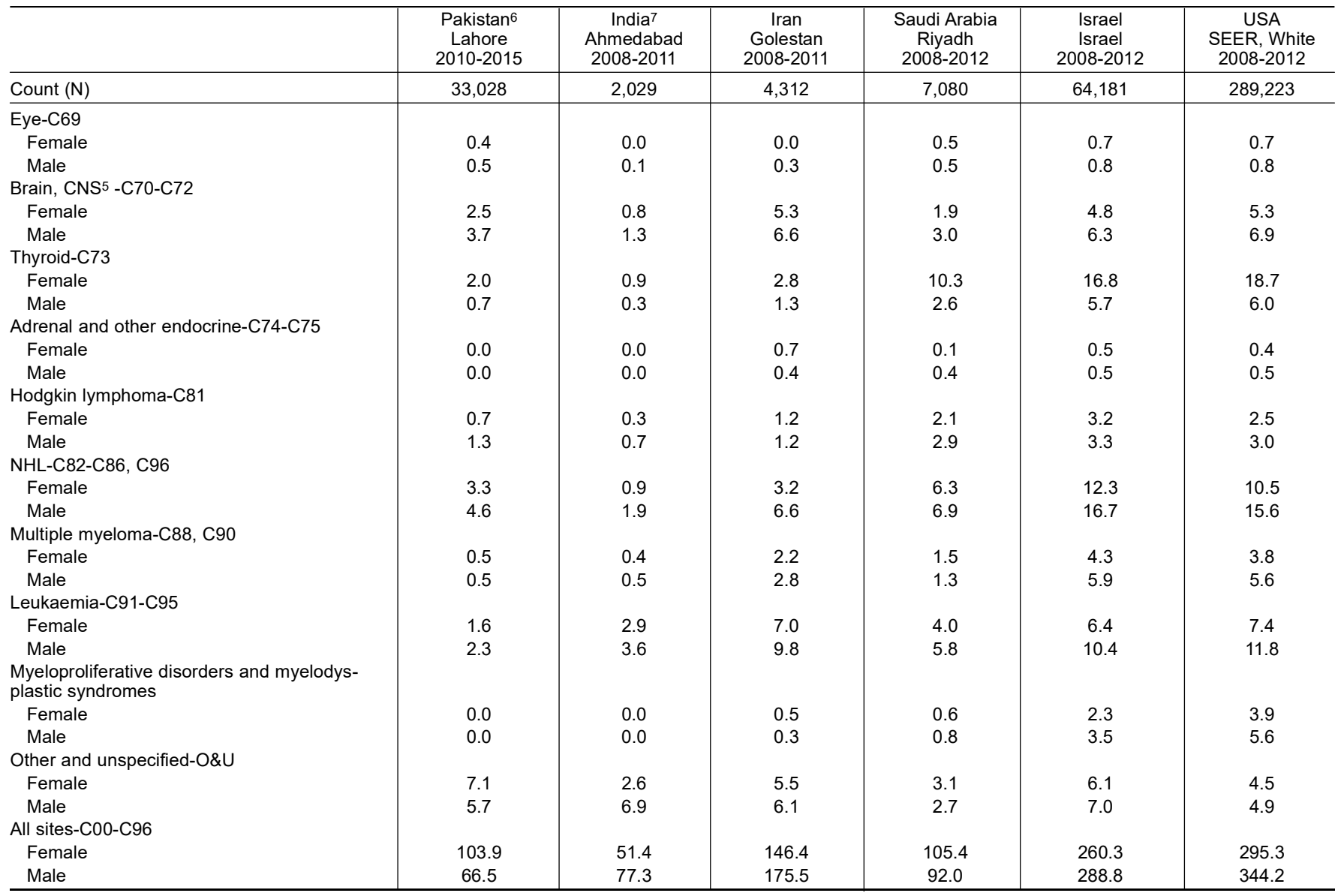

${ }^{1}$ Results for the Lahore district, Pakistan, include C44 skin tumours.

${ }^{2}$ Results for India, Iran, Saudi Arabia, Israel, and USA were obtained from IARC's publication, CI5XI. It includes all sites including C44 skin tumours.

${ }^{3} A$ figure of 0.0 within the table means rates were under 0.1 and - means no statistic was available.

${ }^{4}$ Kidney, etc. includes renal pelvis, ureter, and other \& unspecified urinary organs.

${ }^{5}$ Benign tumors of the brain are not included in the table. However, a total of 103.9 and 66.5 includes an ASIR of 1.7 for females and 1.4 for males, respectively, for benign tumors of the brain

in females representing children (9.4 vs. 6.3, respectively) and adolescents (12.0 vs. 9.0, respectively), but low in adult males (104.1) versus females (168.5).

The ASIRs by cancer site/type are given in Tables I and II. Figure 1 shows high ASIRs for leukaemia among children and for bone and brain/nervous system tumours in adolescents and, the ASIRs for cancers diagnosed in adults, by gender.

The age-specific incidence rates computed for common cancers in females showed that breast cancer peaked markedly (160 per 100,000$)$ between 55 - and 65-years of age, whereas, the incidence rates for cancers of lip/oral cavity, corpus uteri/uterus/placenta, brain/nervous system tumours, and ovary increased slightly with increasing age (Figure 1). Among males, the agespecific incidence rates for prostate cancer continued to rise in the 55+ age group and peaked at 75 (95 per $100,000)$ without showing any decline, whereas, the incidence rates for colo-rectum/anal cancers peaked at 65 -years, but those for lip/oral cavity, brain/nervous system tumours, and lymphoma did not show any significant increase as age increased (Figure 1).

\section{DISCUSSION}

The information related to the vast majority of cancer patients was captured in the Registry as the main hospitals and laboratories of Lahore dealing with patient diagnosis/management are reporting their cases to the Registry. The data are comparable to the data presented by the developed regions of the world in terms of the systems used for classifying/coding the neoplasms, the definition of incidence date applied, and the rules for assessing multiple primaries. However, since no centralised system exists for gathering mortality data in the region, it contributed to the failure to capture the 'death certificate only' cases.

As regards risk factors implicated in the etiology of the disease, tobacco-related cancers have been classified as those of the trachea, bronchus, lung, oral cavity, larynx, pharynx, oesophagus, kidney, bladder, cervix, liver, pancreas, stomach, colon/rectum, and acute myeloid leukaemia. ${ }^{14}$ These cancers together accounted for nearly one-fifth of the cases in females and two-fifths in males in our study (Tables I and II). Based on the World Health Organization's (WHO) 2013 standardised 


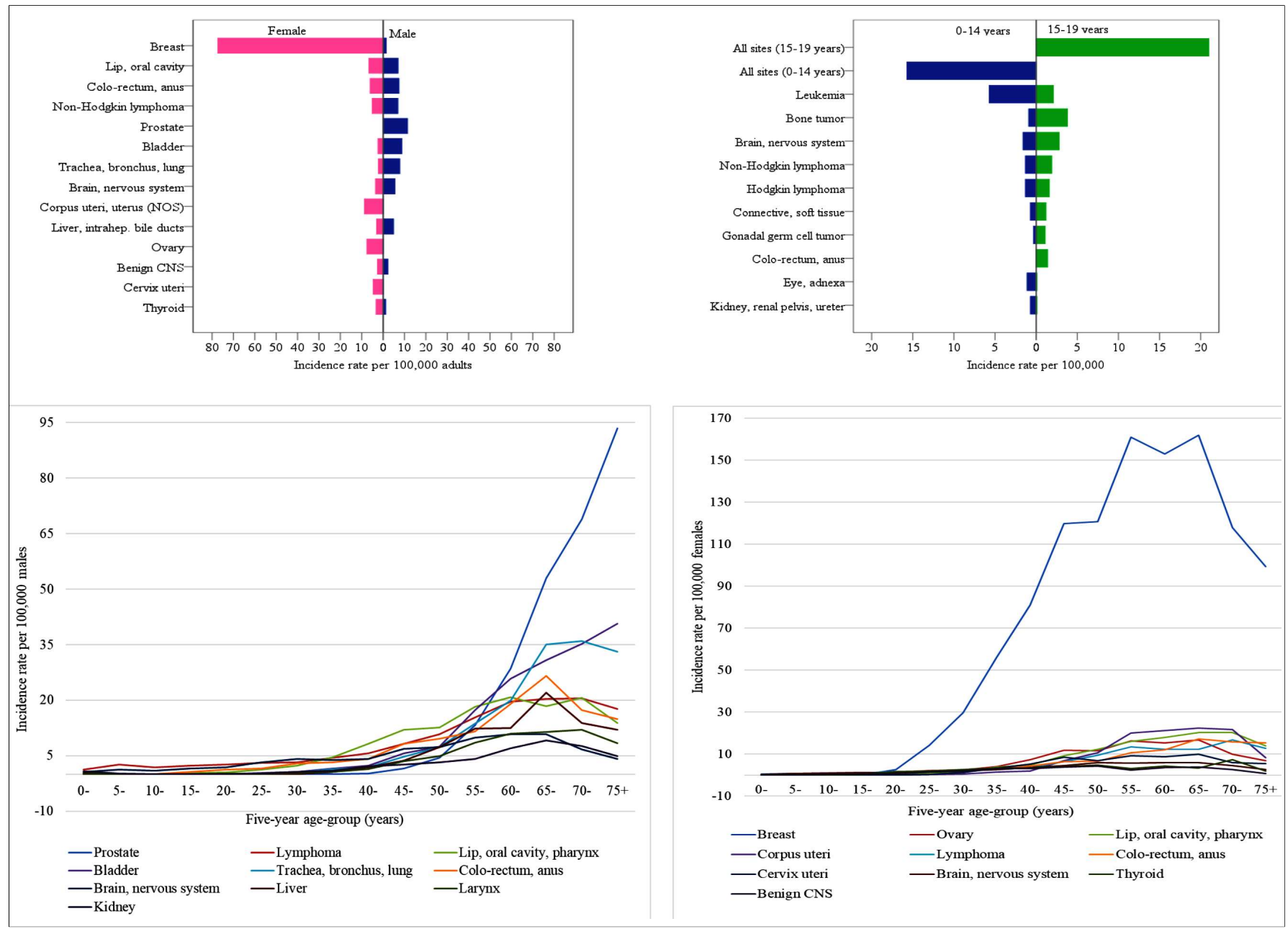

Figure 1: Age-standardised incidence rates per 100,000 population, in children and adolescents, for both sexes combined, among adults, by sex, and age-specific incidence rates, per 100,000 population, for commonly diagnosed cancers among females and males, Lahore, Pakistan, $2010-2015$.

estimate of smoking prevalence, $31.8 \%$ of men, $5.8 \%$ of women, and $19.1 \%$ of Pakistan's adult population currently use tobacco in one form or another, thus partially explaining the occurrence of tobacco-related cancers in our population, especially in males. ${ }^{15}$ According to the WHO, Pakistan is one of the 15 countries worldwide with a heavy burden of tobacco-related conditions. Therefore, it would be worth accelerating different measures to control the manufacture, import, and sale of various tobacco-related products in the country. ${ }^{14}$ However, in our study, the incidence rates for tobacco-related cancers were not very high, thus suggesting that such cases could have been under-reported by the collaborating centres.

Cancer of the stomach did not contribute substantially to the burden in either gender $(<3 \%)$, thus questioning the prevalence of Helicobacter pylori that might increase the risk of stomach cancer. HPV associated cancers as those of the cervix, vagina, and vulva accounted for nearly $3 \%$ of the cancers; of the oral cavity and throat, nearly $4 \%$ in females and nearly $7 \%$ in males; and anal and penile cancers less than $1 \%$ of all cancers (Tables I and II). It has been reported that within Pakistan, almost
12 million people are suffering from hepatitis $\mathrm{B}$ or $\mathrm{C}$, and each year, almost 150,000 new cases are being diagnosed. ${ }^{16}$ In the present study, Hepatocellular Carcinoma (HCC) accounted for nearly $1.5 \%$ of the cases in females and $4 \%$ in males. Since the role of Hepatitis C Virus (HCV) as a risk factor for HCC is well established, and as HCV is a commonly diagnosed infection in Pakistan contributing significantly to the HCV burden globally, the likely role of HCV in the etiology of HCC should be highlighted along with treatment and preventive measures to slow liver damage, and to reduce cancer risk within the population. ${ }^{17}$ Concerning other infections as the Epstein-Barr Virus implicated in the etiology of Hodgkin disease and the Human Immunodeficiency Virus in NHL, extensive studies are needed to elaborate on them as risk factors within our population.

Breast cancer contributed substantially to the cancers recorded in the district (Table I). Studies have shown that the vast majority of cases are sporadic in nature but most of the findings regarding risk factors do not appear to be consistent with one another. ${ }^{7}$ However, young age at menarche, single marital status, nulliparity, late first 
full-term pregnancy, use of oral contraceptives, late menopause, a high body mass index, obesity, and a family history of breast cancer are implicated with an increased risk of this disease. ${ }^{7}$

Further discussion of various risk factors including exposure to ultraviolet radiation and certain chemicals (as asbestos), genetic disorders, and a family history of cancer especially relating to skin cancer and leukaemia, is very extensive and is beyond the scope of this manuscript.

The literature was reviewed to obtain similar reports published on populations adjacent to or close to Lahore or, anywhere else in the country. Other than the KCR's reports on the Karachi South district (1998-2002) published several years ago, 3 the authors were not able to find any reports from Pakistan for the most recent years. However, a comparison of the Lahore district incidence rates was made with the Globocan rates for Pakistan and also the incidence rates reported in Cl-5, Volume XI publication, for selected regions of the world (Table III).18,19 The cancer estimates for Pakistan based on the Globocan 2012 and Globocan 2018 reports have shown that the estimated age-standardised incidence rates are not remarkably different from one another during these two years under consideration. However, the number of incident cases has increased by about $17 \%$, which also means that the cancer burden has gone up considerably over the years.

From the $\mathrm{Cl}-5$ publication, Ahmedabad (India), Golestan (Iran), Riyadh (Saudi Arabia), Israel, and the SEER program were selected. The results for 9-SEER cancer registries studying White population of the US in the 2008-2012 period have shown very high ASIRs as compared to the Lahore district results particularly for cancers of the colo-rectum, trachea/bronchus/lung, breast, prostate, and skin melanoma. This could perhaps be attributed to the regulation requiring case reporting from all facilities/practitioners to state cancer registries, access by the statewide cancer registries to medical records of persons with cancer, and screening and detection practices prevalent in the US apart from differences in the dietary habits, lifestyle factors, and exposure to tobacco. Further comparisons have shown that the ASIRs for Lahore, for all cancers combined were considerably low compared to those for Israel and Golestan, comparable to those for Riyadh; but high compared to the Ahmedabad data. The ASIRs for the Israeli population were, in general, higher than those for Lahore notably for cancers of the GI system, pancreas, lower respiratory tract, female breast, prostate, bladder, brain, thyroid, NHL, multiple myeloma, leukaemia, and skin melanoma. The ASIRs for the Ahmedabad population, compared to the ASIRs for Lahore, were high for cancers of the lip/oral cavity, lower respiratory tract, cervix uteri and leukaemia, low for GI system, prostate, urinary tract, brain, thyroid, Hodgkin lymphoma, and NHL, and comparable with the rest of cancers. The ASIRs for the population of Golestan compared to those for Lahore were considerably high for cancers of the Gastrointestinal (GI) system, pancreas, larynx, lower respiratory tract, prostate, bladder, cervix uteri/other female genital organs, multiple myeloma, and leukaemia, somewhat high for tumours of the ovary, brain/nervous system, thyroid, and $\mathrm{NHL}$, but low for cancers of the oral cavity/salivary glands and female breast cancer. In case of the population of Riyadh, compared to Lahore, the ASIRs for cancers of the GI system, pancreas, lower respiratory tract, corpus uteri, ovary, thyroid, Hodgkin lymphoma, NHL, multiple myeloma, and leukaemia were relatively high, but low for the oral cavity/salivary glands, breast, and cervix uteri. It is worth mentioning here that geographically, Pakistan is located in South Asia and its neighbouring countries include India (South Asia) and Iran (West Asia); Saudi Arabia is in the Middle East, and Israel, also in the Middle East, is located at the eastern end of the Mediterranean Sea. It has been observed that the dietary habits of the inhabitants of Lahore, Golestan, Riyadh, and Israel are alike as a very large majority of the people living in the first three cities are Muslims and consume both meat and a vegetarian diet, while in Israel, Jews consume Kosher food and a Mediterranean diet, but their dietary habits would be different from the people of Ahmedabad, a populous city of India where Hindus constitute nearly $80 \%$ of the population and primarily consume a vegetarian diet. 20 Given that the ASIRs of cancers of the GI system, respiratory tract, and leukaemia were considerably high in Golestan, those of the cervix were high in Ahmedabad, and breast cancer in Lahore and Israel, it would be worth conducting extensive population-based studies to evaluate risk factors implicated in the etiology of the disease, to account for the geographic variations in the epidemiology of cancer.

Finally, as shown in Figure 1, the peaks in the agespecific incidence rates for breast and prostate cancer are similar to what is seen globally.

This is the first time that an extensive 6-year incidence report on cancer has been generated for any district of Pakistan. Pakistan has 150 districts as its administrative units, while the district of Lahore is one of the 36 districts in the province of Punjab. 8 Further, according to the World Bank ${ }^{1}$ and Punjab Growth Strategy, 21 the Lahore district represents nearly $1 / 20^{\text {th }}$ of the population of the country and $1 / 10^{\text {th }}$ of the population of Punjab. Therefore, the generalisability of the results is debatable. However, once reports representing other districts are presented, a comparison can be made to assess the generalisability of the results. Regardless, Lahore is a heavily populated region of Pakistan and the significance of the results cannot be denied. Accordingly, the collection of these statistics is noteworthy as a continuum of care in the region. 


\section{CONCLUSION}

These results warrant expanding cancer registration in the region and sharing statistics with policy-makers to establish hospitals accordingly to manage cancer, along with exploring various risk factors within the population.

\section{DISCLOSURE:}

The PCR is registered under the Societies Registration Act of Pakistan and is also a member of the International Association of Cancer Registries, France. The Registry is being sponsored by the Shaukat Khanum Memorial Trust.

\section{ETHICAL APPROVAL:}

The Institutional Review Board of SKMCH\&RC approved this study.

\section{PATIENTS' CONSENT:}

Individual patient consent was not obtained for this study as a retrospective review of the records was conducted, results were collated and anonymised data presented in the manuscript.

\section{CONFLICT OF INTEREST:}

Authors declared no conflict of interest.

\section{AUTHORS' CONTRIBUTIONS:}

FB: Conceived the idea of the study, designed it, supervised the statistical analysis, did literature search, interpreted the results, drafted the manuscript and finalised it. SM: Did the case finding, indexing and coding of cases, computed the incidence rates and created figures and tables.

MTM, MM, IT, ORC, SN, AA: Contributed to the acquisition of data and are accountable for all aspects of the work in ensuring that questions related to the accuracy or integrity of any part of the work are appropriately investigated and resolved.

\section{REFERENCES}

1. Country Profile [Internet]. Databank.worldbank.org. 2019 [cited 2 September 2019]. Available from: https://databank.worldbank.org/ data/views/reports/reportwidget.aspx?Report_Name=Country Profile\&ld=b450fd57\&tbar=y\&dd=y\&inf=n\&zm=n\&country=PAK.

2. Yusuf A. Cancer Care in Pakistan. Japanese J Clin Oncol 2013; 43:771-5.

3. Curado MP, Edwards B, Shin HR. Pakistan, South Karachi (1998-2002). In: Cancer Incidence in five continents Vol. IX. IARC Scientific Publications No. 160; 2007 [Internet]. Lyon: International Agency for Research on Cancer 2009; [cited 24 December 2019]. Available from: https://www.iarc.fr/wpcontent/uploads/2018/07/CI5vol9.pdf.

4. Nishtar S. Health systems in Pakistan. In: Nishtar S, ed. Choked pipes: Reforming Pakistan's mixed health systems. Karachi: Oxford University Press 2010; p.35-50.

5. Punjab Cancer Registry [Internet]. Punjabcancerregistry. org.pk. 2019 [cited 2 September 2019]. Available from: http://punjabcancerregistry.org.pk/.
6. Badar F, Mahmood S, Yusuf M, Sultan F. Epidemiology of cancers in Lahore, Pakistan, 2010-2012: A cross-sectional study. BMJ Open 2016; 6:e011828.

7. Badar F, Mahmood S. Epidemiology of cancers in Lahore, Pakistan, among children, adolescents and adults, 2010-2012: A cross-sectional study part 2. BMJ Open 2017; 7:e016559.

8. Shah $\mathrm{Q}, \mathrm{Abbas} \mathrm{H}$. Working paper 148. Livelihoods and access to services: An analysis of peri-urban areas of Lahore, Pakistan [Internet]. Islamabad: Sustainable Development Policy Institute; 2015. Available from: https://www.sdpi.org/ publications/files/Livelihoods-and-access-to-services-Ananalysis-of-peri-urban-areas-of-Lahore-Pakistan(W\%20\%20148).pdf.

9. District at a glance Lahore [Internet]. Islamabad: Pakistan Bureau of Statistics-Government of Pakistan; 2015. Available from: http://www.pbs.gov.pk/content/district-glance-lahore.

10. Holden K, ed. ICD-10-CM Expert for Hospitals. The complete official code set. Codes valid October 1, 2015 through September 30, 2016. Salt Lake City, UT: Optum360; 2015. LLC:1-1250.

11. National Cancer Institute, Bethesda, MD, Registre des Cancers de I'Isere, Isere, France, Gerhard Domagk Institut fur Pathologie, Munster Universitat, Munster, Germany, Chirurgische Klinik mit Poliklinik der Universitat Erlangen-Nurnberg, Erlangen, Germany, International Agency for Research on Cancer, Lyon, France, Societa Italiana di Cancerologia, Milan, Italy, University of Chicago, Chicago, IL, USA, European Network of Cancer Registries, and International Society of Pediatric Oncology Working Group, contributors. Morphology. In: Fritz A, Percy C, Jack A, et al. International Classification of Diseases for Oncology. 3rd ed. Geneva: WHO 2000; 27-30.

12. International Association of Cancer Registries, European Network of Cancer Registries and International Agency for Research on Cancer, Working Group. Program for Multiple Primaries-IARC/IACR Multiple Primary Rules. Appendix 3. In: Ferlay J, Burkhard C, Whelan S, eds. Check and conversion programs for cancer registries (IARC/IACR tools for cancer registries). IARC Technical Report No. 42. Lyon: International Agency for Research on Cancer 2005; 38-45.

13. Boyle P, Parkin DM. Chapter 11: Statistical Methods for Registries. In: Jensen OM, Parkin DM, MacLennan R, ed. Cancer Registration: Principles and Methods-IARC Scientific Publication No 95. Lyon IARC; 1991.7.

14. Tobacco control in Pakistan [Internet]. World Health Organization, Geneva. 2015 [cited 2 September 2019]. Available from: https://www.who.int/tobacco/about/partners/ bloomberg/pak/en/.

15. What Causes Cancer? [Internet]. Cancer.org. 2018 [cited 2 September 2019]. Available from: https://www.cancer.org/ cancer/cancer-causes.html.

16. WHO EMRO. Prevention and control of hepatitis | Programmes | Pakistan [Internet]. Emro.who.int. 2019 [cited 2 September 2019]. Available from: http://www.emro.who.int/ pak/programmes/prevention-a-control-of-hepatitis.html.

17. World Health Organization. Health topics | Hepatitis C: simplified curative treatments can drive global scale-up [Internet]. 2018 [cited 2 September 2019]. Available from: http://www.who.int/hepatitis/news-events/hep-c-scale-up/en/.

18. Ferlay J, Ervik M, Lam F. Global Cancer Observatory: Cancer 
Today [Internet]. Lyon: International Agency for Research on Cancer 2018; [cited 24 December 2019]. Available from: http://gco.iarc.fr/today/data/factsheets/ populations/586-pakistanfact-sheets.pdf.

19. World Health Organization. Cancer Incidence in Five Continents-CI5XI [Internet]. Lyon: International Agency for Research on Cancer 2018; [cited 24 December 2019]. Available from: http://ci5.iarc.fr/Cl5-XI/Default.aspx.
20. Ahmedabad [Internet]. 2019. [cited 24 December 2019]. Available from: https://en.wikipedia.org/wiki/Ahmedabad.

21. Punjab Growth Strategy 2018. Accelerating Economic Growth and Improving Social Outcomes. 4.2: Demography and Population Planning-Figure 4. p. 12 [Internet]. Lahore: Planning and Development Department, Government of the Punjab; 2015. Available from: https://www.theigc.org/wp-content/uploads/ 2015/04/Punjab-Growth-Strategy-2018-Full-report.pdf 